\title{
Implantable Cardioverter Defibrillator Therapy in Patients with Cardiac Sarcoidosis
}

\author{
JOSEPH L. SCHULLER, M.D., MATTHEW ZIPSE, M.D., THOMAS CRAWFORD, M.D., \\ FRANK BOGUN, M.D., JOHN BESHAI, M.D., AMIT R. PATEL, M.D., NADERA J. SWEISS, M.D., \\ DUY T. NGUYEN, M.D., RYAN G. ALEONG, M.D., PAUL D. VAROSY, M.D., \\ HOWARD D. WEINBERGER, M.D., and WILLIAM H. SAUER, M.D.
}

From the Section of Cardiac Electrophysiology, University of Colorado, Denver, Colorado, USA; the Section of Cardiac Electrophysiology, University of Michigan, Ann Arbor, Michigan, USA; and the Section of Cardiac Electrophysiology, University of Chicago, Chicago, Illinois, USA

\begin{abstract}
ICD Shocks in Cardiac Sarcoidosis. Background: An implantable cardioverter defibrillator (ICD) is indicated for some patients with cardiac sarcoidosis (CS) for prevention of sudden death. However, there are little data regarding the event rates of ICD therapies in these patients. We sought to identify the incidence and characteristics of ICD therapies in this patient population.

Methods: We performed a cohort study of patients with ICDs at 3 institutions. Cases were those patients with CS and an ICD implanted for primary or secondary prevention of sudden death. Additionally, we included a comparison with historical controls of ICD therapy rates reported in clinical trials evaluating the ICD for primary and secondary prevention of sudden death.
\end{abstract}

Results: Of the 112 CS subjects identified, $36(32.1 \%)$ received appropriate therapies for ventricular tachyarrhythmias (VT) over a mean follow-up period of 29.2 months. VT storm ( $>3$ episodes in 24 hours) occurred in $16(14.2 \%)$ CS subjects. Inappropriate therapies occurred in $13 \mathrm{CS}$ subjects (11.6\%). Covariates associated with appropriate ICD therapies included left ventricular ejection fraction (LVEF) $<55 \%$ (OR 6.52 [95\% CI 2.43-17.5]), right ventricular dysfunction (OR 6.73 [95\% CI 2.69-16.8]), and symptomatic heart failure (OR 4.33 [95\% CI 1.86-10.1]).

Conclusions: In our cohort of patients with CS and ICDs, almost one-third receive appropriate therapies. This may be due to a myocardial inflammatory process leading to increased triggered activity and subsequent scarring leading to reentrant tachyarrhythmias. Adjusted predictors of ICD therapies in this population include left or right ventricular dysfunction. (J Cardiovasc Electrophysiol, Vol. 23, pp. 925-929, September 2012)

cardiac sarcoid, cardiomyopathy, heart failure, implantable cardioverter defibrillator, ventricular tachycardia

\section{Background}

Sarcoidosis is a granulomatous disorder affecting multiple organ systems including the heart with a lifetime prevalence of $0.85-2.4 \%$ depending on ethnicity. ${ }^{1}$ Cardiac involvement is diagnosed antemortem in approximately $5 \%$ of systemic sarcoidosis patients but is seen in up to $25 \%$ of patients with systemic sarcoidosis on autopsy. ${ }^{2}$ The leading causes of death in patients with sarcoidosis are cardiac arrhythmias, progressive heart failure, and progressive respiratory failure. ${ }^{3}$ Cardiac involvement accounts for 13-25\% of all deaths in patients with sarcoidosis. ${ }^{2}$ Sudden death is the most common manifestation of cardiac sarcoidosis, involving more than $60 \%$ of patients. ${ }^{4}$

The mechanism and characteristics of the ventricular arrhythmias in cardiac sarcoidosis have previously been de-

No disclosures.

Address for correspondence: William H. Sauer, M.D., Section of Cardiac Electrophysiology, University of Colorado Hospital, 12401 East $17^{\text {th }}$ Avenue, B136, Aurora, CO 80045, USA. Fax: 720-848-0475; E-mail: william.sauer@ucdenver.edu

Manuscript received 4 October 2011; Revised manuscript received 23 February 2012; Accepted for publication 5 March 2012.

doi: 10.1111/j.1540-8167.2012.02350.x scribed. Cardiac involvement occurs initially with inflammation and granuloma formation followed by scarring. ${ }^{5}$ The initial inflammation can lead to triggered ventricular arrhythmias with subsequent scarring resulting in the substrate for reentrant monomorphic ventricular tachycardia.

Because of the waxing and waning inflammatory nature of the disease process, it is unclear if implantable cardioverter defibrillator (ICD) therapy is effective in the prevention of sudden cardiac death. Several reports have demonstrated the potential for life threatening arrhythmias associated with cardiac sarcoidosis and prophylactic ICD implantation is recommended as a class IIa indication. ${ }^{6}$ Furthermore, it is possible that inflammation can result in ventricular arrhythmias and that the incidence of ICD storm may be more common. We hypothesize that ICD therapies would be common in patients with cardiac sarcoidosis and we hypothesize that VT storm (defined as 3 or more therapies in 24 hours) will also be more common. We sought to identify the incidence and characteristics of ICD therapy in patients with cardiac sarcoidosis.

\section{Methods}

\section{Study Population and Data Collection}

The study cohort consisted of patients referred to the University of Colorado, University of Michigan, and University of Chicago for evaluation of the presence of cardiac 
sarcoidosis. Patients diagnosed with cardiac sarcoidosis who subsequently received an ICD were included in the cohort.

Data collection was performed retrospectively according to a standardized protocol. All patients included in the cohort had biopsy proven extracardiac sarcoidosis.

\section{Criteria for Detection of Cardiac Sarcoidosis}

A modification of the Japanese Ministry of Health and Welfare criteria for diagnosis of cardiac sarcoidosis (to include cardiac MRI and electrophysiologic testing) was used for the diagnosis of cardiac sarcoidosis. Patients were diagnosed with cardiac sarcoidosis if 2 or more of the following studies indicated cardiac involvement:

- ECG and Outpatient Telemetry: Left axis deviation, VT, frequent premature ventricular contractions (PVCs), or abnormal Q waves or ST segment abnormalities (in absence of prior myocardial infarction), bundle branch block or advanced atrio-ventricular (AV) block.

- Echo: Regional wall motion abnormalities, segmental thinning, or dilated left ventricle

- Nuclear: Perfusion defects (without coronary disease) on single photon emission computed tomography or active inflammation observed on positron emission tomography

- Cardiac Catheterization (when available): Elevated intracardiac pressures or low cardiac output

- Cardiac MRI: Delayed intramyocardial contrast enhancement

- Electrophysiologic Study (when available): Inducible monomorphic ventricular tachycardia with up to triple extrastimulation or inducible polymorphic ventricular tachycardia or ventricular fibrillation with up to double extrastimulation; abnormal right ventricular voltage $(<1.5$ $\mathrm{mV}$ ) with electroanatomical mapping

- Histology on Cardiac Biopsy: Nonspecific interstitial fibrosis or cellular infiltration with myocardial biopsy.

\section{ICD Implantation and Clinical Follow-Up}

All patients underwent routine ICD implantation using standard techniques. Perioperative antibiotics were standard, and all patients underwent defibrillation threshold testing at the time of implantation unless contraindicated. If the device was not tested at implant, the patient returned within 3 months for defibrillation threshold testing. Patients were followed with routine wound and device checks within the first 2 weeks after implant, and the devices were interrogated at 3-month intervals afterward. Device interrogation data were stored in the Paceart data software system and in hard copy on site. Device programming was at the discretion of the implanting physician.

When possible, assessment of ventricular function was by cardiac MRI. Once the ICD was implanted or for patients who did not have a cardiac MRI, assessment of ventricular function was by either echo or nuclear imaging. Right ventricular dysfunction was defined as a right ventricular ejection fraction (RVEF) $<45 \%$ by MRI or reported RV dysfunction by echo or nuclear imaging.

\section{Classification of ICD Therapies}

ICD therapies were reviewed and classified based upon the stored device electrograms and clinical scenario by an electrophysiologist. Appropriate therapies were defined as device delivered anti-tachycardia pacing (ATP) or shocks for
TABLE 1

Clinical Characteristics of Patients with Cardiac Sarcoidosis (CS) and an Implantable Cardioverter Defibrillator (ICD)

\begin{tabular}{lc}
\hline Cardiac Sarcoidosis Cohort & \\
\hline $\mathrm{N}=112$ & 112 \\
Age (years) & $52.6 \pm 11.4$ \\
Male gender & $63(56.3 \%)$ \\
LVEF mean & $44.9 \pm 17.1 \%$ \\
RV dysfunction & $54(48.2 \%)$ \\
NYHA class II-IV & $48(42.9 \%)$ \\
CAD & $5(4.5 \%)$ \\
Diabetes & $22(19.6 \%)$ \\
HTN & $43(38.4 \%)$ \\
Hyperlipidemia & $31(27.7 \%)$ \\
Renal insufficiency & $22(19.6 \%)$ \\
Complete heart block & $17(15.2 \%)$ \\
Right bundle branch block & $30(26.8 \%)$ \\
\hline
\end{tabular}

VT or VF. Inappropriate therapies were ATP or shocks resulting from supraventricular arrhythmias (including atrial fibrillation and flutter), $\mathrm{T}$ wave oversensing, or lead noise. ICD storm was defined as 3 or more appropriate ICD therapies in a 24-hour period.

\section{Statistical Analysis}

Results are expressed as mean \pm standard deviation. The Pearson chi-square test was used to compare clinical characteristics between patients with and without cardiac sarcoidosis. The one-way analysis of variance (ANOVA) was used for continuous covariates. Multivariable logistic regression analysis was used to adjust for possible confounding. The variables included in the multivariable model were variables that affected the crude odds ratio (OR) by more than $10 \%$. The variables used in the multivariable model were age, sex, left ventricular ejection fraction, right ventricular dysfunction, New York Heart Association functional class, history of hypertension, coronary disease, diabetes mellitus, or renal insufficiency. Statistical analyses were performed using the SPSS (version 18.0; SPSS Inc, Chicago, IL, USA) statistical software program, and statistical significance was defined as a two-sided $\mathrm{P}$ value less than 0.05 .

\section{Results}

\section{Clinical Characteristics of Study Cohort}

Table 1 displays the clinical characteristics of the study cohort. We identified 112 ICD patients with cardiac sarcoidosis that were followed for a mean of 29.2 months. The mean age of the cohort was 52.6 years, and 53\% were men. The mean left ventricular ejection fraction was $44.9 \%$ and right ventricular dysfunction was noted in $48 \%$. There were 5 patients with coronary artery disease, 22 with diabetes mellitus, 43 with hypertension, 31 with hyperlipidemia, and 22 with chronic renal insufficiency (Tables 1 and 2). Seventeen patients had a history of complete heart block, and 30 patients demonstrated right bundle branch block. There were 6 patient deaths among the cohort. Four patients underwent cardiac transplantation.

\section{Incidence of Appropriate and Inappropriate ICD Therapy}

Of the 112 subjects with cardiac sarcoidosis, 36 (32.1\%) received appropriate therapies for ventricular tachyarrhythmias (VT). ICD storm, defined as 3 or more appropriate ICD 
TABLE 2

Comparison of Clinical Characteristics of Patients with CS with and without ICD Therapies

\begin{tabular}{|c|c|c|c|c|c|c|}
\hline Cardiac Sarcoidosis Cohort & No Therapies & Any Therapy & P Value & Appropriate Therapy & P Value & \\
\hline $\mathrm{N}=112$ & 66 & $46(41.1 \%)$ & & $36(32.1 \%)$ & & \\
\hline Age (years) & $55.1 \pm 11.3$ & $48.9 \pm 10.7$ & 0.004 & $49.5 \pm 11.1$ & 0.052 & \\
\hline Male gender & $34(51.5 \%)$ & $29(63 \%)$ & 0.25 & $26(72.2 \%)$ & 0.025 & \\
\hline LVEF mean & $50.6 \pm 14.1$ & $37.3 \pm 18$ & $<0.01$ & $32.2 \pm 16.5$ & $<0.01$ & \\
\hline RV dysfunction & $24(36.4 \%)$ & $30(65.2 \%)$ & $<0.01$ & $28(77.8 \%)$ & $<0.01$ & \\
\hline NYHA class II-IV & $20(30.3 \%)$ & $28(60.9 \%)$ & $<0.01$ & $24(66.7 \%)$ & $<0.01$ & \\
\hline CAD & $4(6.1 \%)$ & $1(2.2 \%)$ & 0.65 & $1(2.8 \%)$ & 1 & \\
\hline Diabetes & $11(16.7 \%)$ & $11(23.9 \%)$ & 0.35 & $9(25 \%)$ & 0.32 & \\
\hline HTN & $28(42.4 \%)$ & $15(32.6 \%)$ & 0.33 & $10(27.8 \%)$ & 0.15 & \\
\hline Hyperlipidemia & $18(27.3 \%)$ & $13(28.3 \%)$ & 1 & $8(22.2 \%)$ & 0.5 & \\
\hline Renal insufficiency & $13(19.7 \%)$ & $9(19.6 \%)$ & 1 & $9(25 \%)$ & 0.32 & \\
\hline Complete heart block & $10(15.2 \%)$ & $8(17.4 \%)$ & 1 & $7(19.4 \%)$ & 0.41 & \\
\hline Right bundle branch block & $21(31.8 \%)$ & $10(21.7)$ & 1 & $9(25 \%)$ & 0.32 & \\
\hline Cardiac Sarcoidosis Cohort & \multicolumn{3}{|c|}{ Inappropriate Therapy } & P Value & VT Storm & P Value \\
\hline $\mathrm{N}=112$ & \multicolumn{3}{|c|}{$13(11.6 \%)$} & & $16(14.2 \%)$ & \\
\hline Age (years) & \multicolumn{3}{|c|}{$49.2 \pm 11.1$} & 0.25 & $47 \pm 9.4$ & 0.035 \\
\hline Male gender & \multicolumn{3}{|c|}{$5(38.5 \%)$} & 0.24 & $12(75 \%)$ & 0.17 \\
\hline LVEF mean & \multicolumn{3}{|c|}{$50.2 \pm 14$} & 0.24 & $34 \pm 16.8$ & $<0.01$ \\
\hline RV dysfunction & \multicolumn{3}{|c|}{$7(53.8 \%)$} & 0.77 & $12(75 \%)$ & 0.029 \\
\hline NYHA class II-IV & \multicolumn{3}{|c|}{$4(30.8 \%)$} & 0.39 & $9(56.3 \%)$ & 0.28 \\
\hline CAD & \multicolumn{3}{|c|}{0} & 1 & 0 & 1 \\
\hline Diabetes & \multicolumn{3}{|c|}{$3(23.1 \%)$} & 0.72 & $4(25 \%)$ & 0.52 \\
\hline HTN & \multicolumn{3}{|c|}{$4(30.8 \%)$} & 0.76 & $6(37.5 \%)$ & 0.55 \\
\hline Hyperlipidemia & \multicolumn{3}{|c|}{$5(38.5 \%)$} & 0.34 & $3(18.8 \%)$ & 1 \\
\hline Renal insufficiency & \multicolumn{3}{|c|}{$2(15.4 \%)$} & 1 & $3(18.8 \%)$ & 1 \\
\hline Complete heart block & & 1 & $5(31.3 \%)$ & 0.54 \\
\hline Right bundle branch block & & $3(23.1$ & & 0.72 & $5(31.3 \%)$ & 0.54 \\
\hline
\end{tabular}

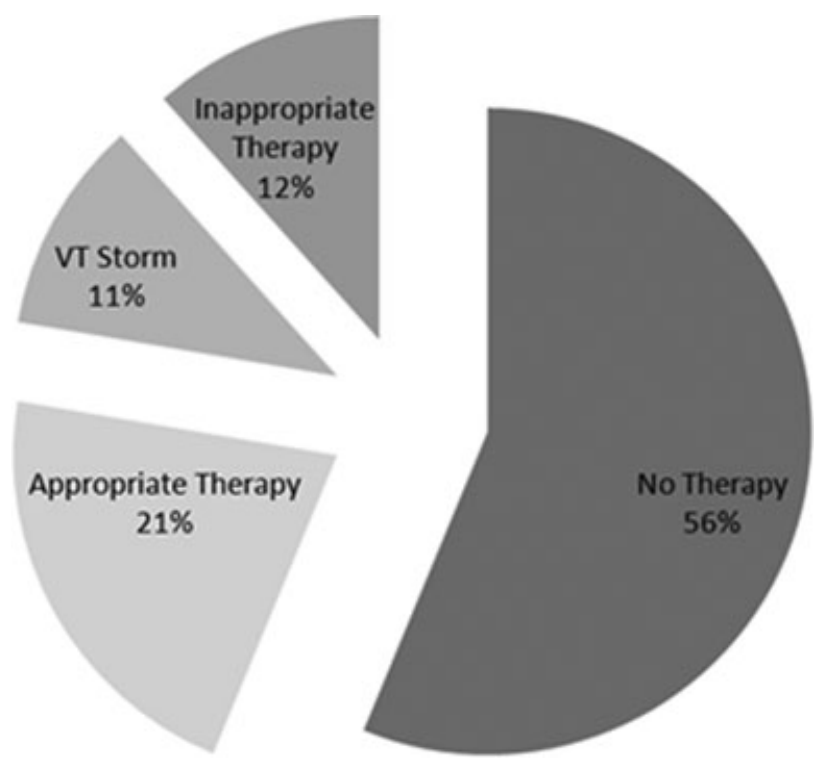

Figure 1. Incidence of ICD therapies, including VT storm, in patients with cardiac sarcoidosis.

therapies in 24 hours, occurred in 16 of the 112 (14.3\%) of the cardiac sarcoidosis patients. Thirteen patients in the cohort received inappropriate therapy (11.6\%) (Fig. 1).

In an unadjusted analysis there were 3 predictors of appropriate ICD therapies identified. Left ventricular dysfunction as assessed by either MRI or echocardiogram was associated with an OR of 6.52 (95\% CI 2.43-17.5, P $<0.01)$. Right ventricular dysfunction as assessed by MRI or echocardiogram was associated with an OR of 6.73 (95\% CI 2.69-16.8, P < 0.01). Symptomatic heart failure (New York Heart Association functional class II-IV) was associated with an OR of 4.33 (95\% CI 1.86-10.1, $\mathrm{P}<0.01$ ). Of the 36 patients who received appropriate ICD therapy, only 1 patient had normal right and left ventricular function.

After multivariable adjustment, left and right ventricular dysfunction remained significant predictors of future ICD therapies (adjusted OR for LV dysfunction 6.52 [95\% CI 2.43-17.5, $\mathrm{P}<0.01$ ], adjusted OR for RV dysfunction 5.64 [95\% CI 2.19-14.6, $\mathrm{P}<0.01])$. After multivariable adjustment, symptomatic heart failure was no longer significantly associated with appropriate ICD therapies (OR 1.89 [95\% CI 0.69-5.15], $\mathrm{P}=0.21$ ).

\section{ICD Storm in Patients with Cardiac Sarcoidosis}

ICD storm, defined as 3 or more appropriate ICD therapies in 24 hours, occurred in 16 of the $112(14.3 \%)$ of the cardiac sarcoidosis patients. Predictors of ICD storm included left ventricular dysfunction (OR 6.71 [95\% CI 1.45-31.2], $\mathrm{P}=$ $0.015)$ and right ventricular dysfunction (OR 3.86 [95\% CI 1.16-12.8], $\mathrm{P}=0.03$ ).

\section{Prediction of Ventricular Arrhythmias in Patients with Cardiac Sarcoidosis and Primary versus Secondary Prevention Devices}

Of the 112 patients with cardiac sarcoidosis and an ICD, $83(74.1 \%)$ were implanted for primary prevention. Among this subset, $23(27.7 \%)$ received appropriate ICD therapy for VT or VF over a mean follow-up period of 30.6 months. Of 
the primary prevention subset, no patients with normal right and left ventricular function received an appropriate therapy. The mean left ventricular ejection fraction among the secondary prevention subset was $40.6 \%$ and among the primary prevention subset was $46.4 \%$. Right ventricular dysfunction was present in $53.6 \%$ of the secondary prevention subset and in $46.4 \%$ of the primary prevention subset. In comparison with the patients with primary prevention devices, a previous history VT/VF was not a significant predictor of appropriate ICD therapies (OR 1.36 [CI 0.53-3.48]).

\section{Discussion}

\section{Study Results}

This study details our experience with cardiac sarcoidosis and ICDs at 3 institutions with a relatively large population of patients with cardiac sarcoidosis. We found that ICD therapies are frequent in patients with cardiac sarcoidosis. Ventricular dysfunction appears to be a strong predictor of ventricular arrhythmias. This is likely a reflection of a more advanced disease state with either transient inflammation with associated contractile dysfunction, or ventricular scarring with associated reentrant arrhythmias.

Further, the rate of ICD therapies in our cohort of cardiac sarcoidosis is greater than reported rates from historical controls. In the large randomized trials PREPARE and SCDHeFT, annualized ICD therapy rates of $5.4 \%$ and $5.1 \%$ were reported, respectively. ${ }^{7,8}$ In the secondary prevention trial SMASH-VT, an annualized ICD therapy rate of $16.5 \%$ was reported. ${ }^{9}$ The annualized appropriate ICD therapy rate in our cohort was $13.2 \%$. Among the primary prevention subgroup the annualized ICD therapy rate was $10.7 \%$, and among the secondary prevention subgroup the annualized ICD therapy rate was $21 \%$.

Reports of ICD therapy rates from other infiltrative cardiomyopathies are also similarly high. ICD therapy rates in arrhythmogenic right ventricular cardiomyopathy range from $24 \%$ to $74 \%$ at 5 years. ${ }^{10}$

We also observed that ICD storm was common in our cohort of cardiac sarcoidosis, occurring in $14.3 \%$ of the cardiac sarcoidosis patients.

\section{Use of Primary Prevention ICDs in Patients with Cardiac Sarcoidosis}

Cardiac sarcoidosis is a rare disease that is often difficult to diagnose and there is little known regarding the potential role of the implantable defibrillator for prevention of sudden death. In patients with sarcoidosis, ICD therapy can be used as a component of an aggressive primary prevention strategy when cardiac involvement is established. For sarcoidosis patients who present with sustained ventricular arrhythmias and are diagnosed with cardiac involvement, ICD implantation is standard practice. To date, there have been no prospective studies comparing ICD therapy with antiarrhythmic drugs or immunosuppression. This report is the largest experience with a primary and secondary prevention ICD strategy. As one might expect, the rate of ICD therapies is very high among the patients with a secondary prevention device. Interestingly, even among patients with a primary prevention device the incidence of appropriate ICD therapies was $11.3 \%$ annually. This is a higher annualized rate than reported in other large primary prevention ICD trials.
The reported annualized ICD therapy rate among patients with arrhythmogenic right ventricular cardiomyopathy and primary prevention device is $4.9 \% .^{11}$

\section{Potential Mechanisms for Ventricular Tachycardia}

As described in the introduction, there is evidence for multiple mechanisms for VT in cardiac sarcoidosis. The inflammatory nature of the granulomas in sarcoidosis may lead to increased automatic ventricular activity. The subsequent scar formation is then the substrate for reentrant ventricular arrhythmias. This combination of underlying mechanisms makes treating the arrhythmias challenging and is possibly the reason for the relatively high rate of therapies observed in this cohort.

\section{Potential Limitations}

The limitations of observational research must be considered in this study. One such limitation involves the certainty of the cardiac sarcoidosis diagnosis in our cohort. We used a clinical diagnostic criteria scheme that has not been correlated with autopsy studies proving a histologic diagnosis. In addition, there remains the possibility of false positive radiological studies leading to an inaccurate diagnosis. However, we believe that corroborated abnormal cardiac findings in patients with extra-cardiac sarcoidosis strongly suggest cardiac involvement. If there were a misclassification bias, then this bias would be toward a lower rate of appropriate ICD therapy as patients without cardiac sarcoidosis would not be expected to have ventricular arrhythmias.

Another limitation of the study involves the relatively small number of events reducing our ability to evaluate potential confounders in the multivariable analysis. Our comparison to historical control groups is not matched by age, gender, or clinical features; however, we believe that an unmatched comparison is useful to clinicians who may be faced with the clinical question of ICD implantation in these patients.

Data regarding ICD programming, VT cycle length, or treatment for ventricular fibrillation were not available for all patients across the 3 study sites and were not included in our analysis. Similarly, data regarding ICD implantation complication rates, background medical therapy, and nonsustained ventricular arrhythmias were not uniformly collected and therefore were not included in the analysis. However, while all of these covariates may influence the rate of appropriate ICD therapy, we believe that these variables alone do not explain the high rate of ICD therapy seen in our cohort. The clinicians responsible for the care of these patients at the 3 participating sites are all experienced electrophysiologists who routinely program devices to prevent unnecessary ICD therapy.

\section{Conclusions}

ICD therapies in patients with cardiac sarcoidosis are common. The rates of appropriate and inappropriate therapies are similar to those seen in other populations of highrisk patients. The notable exception of VT storm appears to be more common in the cardiac sarcoidosis cohort. Based on the findings of this study we recommend an aggressive prospective screening approach for patients with biopsy proven extra-cardiac sarcoidosis in whom cardiac involvement is suspected. 


\section{References}

1. Chapelon-Abric C, de Zuttere D, Duhaut P, Veyssier P, Wechsler B, Huong DL, de Gennes C, Papo T, Blétry O, Godeau P, Piette JC: Cardiac sarcoidosis: A retrospective study of 41 cases. Medicine (Baltimore) 2004;83:315-334.

2. Silverman KJ, Hutchins GM, Bulkley BH: Cardiac sarcoid: A clinicopathologic study of 84 unselected patients with systemic sarcoidosis. Circulation 1978;58:1204-1211.

3. Perry A, Vuitch F: Causes of death in patients with sarcoidosis. A morphologic study of 38 autopsies with clinicopathologic correlations. Arch Pathol Lab Med 1995;119:167-172.

4. Roberts WC, McAllister HA Jr, Ferrans VJ: Sarcoidosis of the heart. A clinicopathologic study of 35 necropsy patients (group 1) and review of 78 previously described necropsy patients (group 11). Am J Med 1977;63:86-108.

5. Ferrans VJ, HibbsS RG, Black WC, Walsh JJ, Burch GE: Myocardial degeneration in cardiac sarcoidosis: Histochemical and electron microscopic studies. Am Heart J 1965;69:159-172.

6. Epstein AE, Dimarco JP, Ellenbogen KA, Estes NA 3rd, Freedman RA, Gettes LS, Gillinov AM, Gregoratos G, Hammill SC, Hayes DL, Hlatky MA, Newby LK, Page RL, Schoenfeld MH, Silka MJ, Stevenson LW, Sweeney MO: ACC/AHA/HRS 2008 Guidelines for Device-Based Therapy of Cardiac Rhythm Abnormalities: A report of the American College of Cardiology/American Heart Association Task Force on Practice Guidelines (Writing Committee to Revise the ACC/AHA/NASPE 2002 Guideline Update for Implantation of Cardiac Pacemakers and Antiarrhythmia Devices): Developed in collaboration with the American Association for Thoracic Surgery and Society of Thoracic Surgeons. Circulation 2008;117:e350-e408.
7. Wilkoff BL, Williamson BD, Stern RS, Moore SL, Lu F, Lee SW, Birgersdotter-Green UM, Wathen MS, Van Gelder IC, Heubner BM, Brown ML, Holloman KK: Strategic programming of detection and therapy parameters in implantable cardioverter-defibrillators reduces shocks in primary prevention patients: Results from the PREPARE (Primary Prevention Parameters Evaluation) study. J Am Coll Cardiol 2008;52(7):541-550.

8. Bardy GH, Lee KL, Mark DB, Poole JE, Packer DL, Boineau R, Domanski M, Troutman C, Anderson J, Johnson G, McNulty SE, Clapp-Channing N, Davidson-Ray LD, Fraulo ES, Fishbein DP, Luceri RM, Ip JH: Amiodarone or an implantable cardioverterdefibrillator for congestive heart failure. N Engl J Med 2005;352:225237.

9. Reddy VY, Reynolds MR, Neuzil P, Richardson AW, Taborsky M, Jongnarangsin K, Kralovec S, Sediva L, Ruskin JN, Josephson ME: Prophylactic catheter ablation for the prevention of defibrillator therapy. N Engl J Med 2007;357:2657-2665.

10. Wichter T, Paul M, Wollmann C, Acil T, Gerdes P, Ashraf O, Tjan TD, Soeparwata R, Block M, Borggrefe M, Scheld HH, Breithardt G, Böcker D: Implantable cardioverter/defibrillator therapy in arrhythmogenic right ventricular cardiomyopathy: Single-center experience of long-term follow-up and complications in 60 patients. Circulation 2004;109:1503-1508

11. Corrado D, Calkins H, Link MS, Leoni L, Favale S, Bevilacqua M, Basso C, Ward D, Boriani G, Ricci R, Piccini JP, Dalal D, Santini M, Buja G, Iliceto S, Estes NA 3rd, Wichter T, McKenna WJ, Thiene G, Marcus FI: Prophylactic implantable defibrillator in patients with arrhythmogenic right ventricular cardiomyopathy/dysplasia and no prior ventricular fibrillation or sustained ventricular tachycardia. Circulation 2010;122:1144-1152. 